\title{
Fractional-order models: The case study of the supercapacitor capacitance measurement
}

\author{
M. LEWANDOWSKI ${ }^{1 *}$ and M. ORZYŁOWSKI ${ }^{2}$ \\ ${ }^{1}$ Warsaw University of Technology, Faculty of Electrical Engineering, 1 Politechniki Sq., 00-661 Warszawa, Poland \\ ${ }^{2}$ University of Social Sciences, Information Technology Institute, 9 Sienkiewicza St., 90-113 Łódź, Poland
}

\begin{abstract}
At the beginning of the paper, the fractional calculus is briefly presented. Then, the models of dielectric relaxation in supercapacitors are described. On the basis of the Cole-Cole model, a fractional-order model of supercapacitor impedance is formulated. The frequency characteristics of selected supercapacitors and their voltage response to a current step are assumed as a basis for the analysis of their dynamics. An example of the fractional dynamic model application was used for the critical assessment of the IEC standard recommendation on the conditions of supercapacitor capacitance measurements. The presented study shows some imperfections of the IEC standard recommendations, which probably result from the use of an inaccurate dynamics model. At the end of the paper, the authors propose a solution to this problem by changing the measurement conditions and introducing a concept of dynamic capacitance. The conclusions of the paper indicate that the models of fractional-order dynamics may be useful not only for the control purposes but also in other domains.
\end{abstract}

Key words: fractional calculus, supercapacitor model, capacitance measurement, IEC standard.

\section{Introduction}

The dynamics of systems can, in general, be described by integer order differential equations. The equations can be formulated as the result of identification of a system treated as a black box, but they are often associated with the description of physical phenomena. In some cases, the integer order models are not sufficiently accurate or effective, which often takes place in the description of physical phenomena, such as heat conduction, electrochemical phenomena, etc. [1-4]. Recently, some works were conducted on the fractional-order dynamics models destined mainly for control purposes $[1,2]$. As a result of these works, such calculation tools are generally available $[1,5]$ that facilitate practical calculations without the intricate description connected with fractional calculus.

The paper presents the application of fractional calculus for the solution of a metrological problem on the example of supercapacitor capacitance measurement. Supercapacitors are an important group of devices storing electric energy. They are used to power both various mobile low-power devices, as well as high-power ones, such as electric vehicles. Although they have lower energy density than the batteries, they have a higher power density. As a result, their charging and discharging times are shorter, and they can be used e.g. for effective storage of breaking energy and to supply the driving energy to a vehicle during acceleration $[19,20]$. The dynamics of supercapacitors can be described by integer order models $[6,8]$, as well as by fractional ones, e.g. [8, 14-16].

*e-mail: miroslaw.lewandowski@ee.pw.edu.pl

Manuscript submitted 2016-06-07, revised 2016-09-05, 2017-01-13 and 2017-02-13, initially accepted for publication 2017-02-26, published in August 2017.
At the beginning, after some information about fractional calculus, the models of dielectric relaxation in supercapacitors are described. On this basis, a fractional-order model of supercapacitor impedance is formulated. In accordance with the recommendation of the IEC standard concerning the requirements for supercapacitor capacitance measurement [21], the analyses of frequency characteristics of selected supercapacitor samples and their voltage responses to current steps were made. The study shows the imperfection of the IEC standard recommendations, which probably results from the use of an inaccurate dynamics model. At the end of the paper, the authors propose a solution to this problem by changing the measurement conditions and by introducing a concept of dynamic capacitance.

\section{Fractional-order dynamic models}

As mentioned, most dynamic systems are described by the integer order models. The dynamics is described in many ways. One of the widely used methods is to employ the differential equation of general form:

$$
\begin{aligned}
& a_{n} \frac{d^{n} y}{d t^{n}}+a_{n-1} \frac{d^{n-1} y}{d t^{n-1}}+\cdots a_{1} \frac{d y}{d t}+a_{0} y=b_{m} \frac{d^{m} u}{d t^{m}}+ \\
& +b_{m-1} \frac{d^{m-1} u}{d t^{m-1}}+,,, b_{1} \frac{d u}{d t}+b_{0} u
\end{aligned}
$$

where $u(t)$ is the input variable, $y(t)$ is the output variable, and $n$ and $m$ are natural numbers. Another common description is the transfer function:

$$
G(s)=\frac{Y(s)}{U(s)}=\frac{b_{m} s^{m}+b_{m-1} s^{m-1} \ldots b_{1} s+b_{0}}{a_{n} s^{n}+a_{n-1} s^{n-1}+\cdots a_{1} s+a_{0}}
$$


where $U(s)$ is the Laplace transform of input signal, $L\{u(t)\}$ and $Y(s)$ is the transform of output signal $L\{y(t)\}$. The output signal is determined by the inverse Laplace transform:

$$
y(t)=L^{-1}\{G(s) U(s)\}
$$

The parameters $a_{i}$ and $b_{i}$ of transfer function of a real system are, in most cases, identified on the basis of the frequency characteristics that can be approximated by the Fourier transform $F(j \omega)$, which is associated with the Laplace transform by replacing the operator $j \omega$ with the operator $s$.

Some systems, e.g. electrochemical, thermal, mechanical, and hydraulic systems, etc., require the use of relatively highorder differential equations for precise description by the integer order models of dynamics [6]. In many cases it is possible to reduce the number of parameters in the description of the dynamics, while improving the modelling accuracy by using the fractional-order differential equations. As mentioned, such models can often be directly associated with physical phenomena.

The basic problems of the fractional calculus are described in numerous publications, e.g. $[1,5]$. The fractional-order differential equations are based on Grünwald-Letnikov, Riemann-Liouville, and Caputo definitions. Using the fractional-order operator $\mathrm{D} \alpha$, the dynamics of the system can be described as [1]:

$$
\begin{aligned}
& a_{n} D^{\alpha_{n}} y+a_{n-1} D^{\alpha_{n-1}} y+\cdots+a_{1} D^{\alpha_{1}} y+a_{0} D^{\alpha_{0}} y= \\
& =b_{m} D^{\beta_{m}} u+\cdots+b_{1} D^{\beta_{1}} u+b_{0} D^{\beta_{0}} u
\end{aligned}
$$

In the above equation, the coefficients $a_{k}, b_{k}$, and the exponents $\alpha_{k}$ and $\beta_{k}$ are real numbers.

Analogically to the transfer function (2), the dynamics of a system can be described by the fractional-order transfer function:

$$
G(s)=\frac{b_{m} s^{\beta m}+b_{m-1} m-1+\cdots+b_{1} s^{\beta_{1}}+b_{0} s^{\beta_{0}}}{a_{n} s^{\alpha} n+a_{n-1} s^{\alpha} n-1+\cdots+a_{1} s^{\beta_{1}}+a_{0} s^{\alpha}}
$$

The calculation tools based on fractional calculus are generally available. One of them is the FOTF toolbox for MATLAB $[1,5]$. It is based on the dynamics description in the form of transfer function (5). It enables to form the series, parallel, and feedback connections of dynamic blocks and to simulate time response.

\section{Dynamic characteristics of supercapacitors}

The supercapacitor is schematically shown in Fig. 1 [7]. The electric charge is stored at the electrode/electrolyte double-layer interface. The roles of the electrodes are played by the porous material (3) and the surrounding electrolyte (5). The dielectric layers are formed by the ions adsorbed at the interface between the porous electrodes and the electrolyte. The electric parameters of supercapacitors are closely related to the physical phenomena in the dielectric layers, especially to

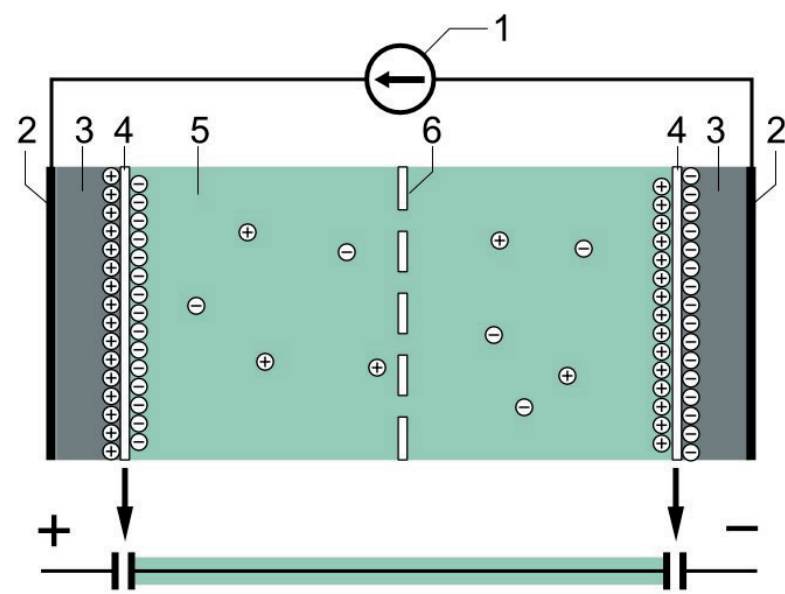

Fig. 1. Typical structure of a supercapacitor: 1) Power source, 2) collector, 3 ) polarized electrode, 4) Helmholtz double layer, 5) electrolyte having positive and negative ions, 6) separator [7]

the dielectric relaxation phenomenon $[8,10,11]$. The parameters also significantly depend on the porosity of the electrodes $[8,9]$.

The capacitor impedance is generally represented by the equivalent circuit diagram, presented in Fig. 2. This scheme includes the serial resistance $R_{c}$, the parallel resistance $R_{u}$, and the series inductance $L$. For a typical capacitor, the capacitance, shown in Fig. 2, may generally be regarded as a constant function of frequency. Although in the description of the supercapacitor impedance, the variation of capacitance with frequency [22] must also be taken into account.

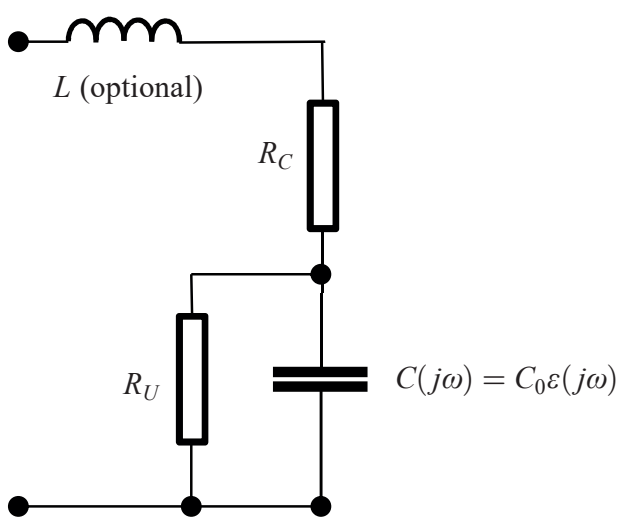

Fig.2. An equivalent circuit of a supercapacitor

The modelling of the supercapacitor impedance using integer order models is associated with a relatively high order $[6,8,17]$. Linking the supercapacitor impedance with its physical parameters leads to description by fractional order equations. The description can be based on the RC distributed model $[8,12]$. The mathematical description of the impedance includes, in this case, the hyperbolic function. This makes the impedance analysis difficult, but this inconvenience can be 
eliminated by applying the Padé approximation [11]. The result is a simpler model described by fractional order equations.

The fractional order model can also be created by including in its structure the Warburg element [8], which models the diffusion processes in dielectric spectroscopy. The most general description of the dynamic phenomena in supercapacitors is obtained by the use of Havriliak-Negami dielectric relaxation model [8].

This paper focuses on the influence of the dielectric relaxation phenomenon on the dynamic properties of supercapacitors. Typical characteristic relaxation time constants are in the range of a few to tens of seconds [11]. This results in the fact that a significant relaxation phenomenon may occur in the frequency range of approx. $0.001 \mathrm{~Hz}$ to several tens of $\mathrm{Hz}$. Therefore, the inductance $L$ can be omitted in these considerations, because its influence can only be seen at a very high frequency.

The phenomenon of dielectric relaxation in supercapacitors for the ideal case is described by the Debye model. In practice, the experimental modifications of this model [13] are used. The description of dielectric permittivity expressed in complex numbers is formulated in the Havriliak-Negami model:

$$
\varepsilon_{H N}(j \omega)=\varepsilon_{\infty}+\frac{\varepsilon_{S}-\varepsilon_{\infty}}{\left[1+(j \omega T)^{\delta}\right]^{\gamma}}, 0 \leq \delta<1,0 \leq \gamma<1,
$$

where:

$\omega-$ angular frequency,

$\varepsilon_{\infty}-$ permittivity at the high frequency band,

$\varepsilon_{S}-$ static, low frequency permittivity,

$T$ - the characteristic relaxation time,

$\gamma, \delta-$ empirically-chosen coefficients.

For the Debey model in the equation (6), the coefficients are $\gamma=1$ and $\delta=1$. For the condition of $\gamma=1$ and $\delta<1$, the Cole-Cole model is obtained:

$$
\varepsilon_{C C}(j \omega)=\varepsilon_{\infty}+\frac{\varepsilon_{S}-\varepsilon_{\infty}}{1+(j \omega T)^{\delta}} \quad 0 \leq \delta<1,
$$

while for $\gamma<1$ and $\delta=1$, the Cole-Davidson model is obtained:

$$
\varepsilon_{C D}(j \omega)=\varepsilon_{\infty}+\frac{\varepsilon_{S}-\varepsilon_{\infty}}{(1+j \omega T)^{\gamma}} \quad 0 \leq \gamma<1,
$$

In supercapacitors, $\varepsilon_{s}>\varepsilon_{\infty}$ [22], therefore herein we assume $\varepsilon_{\infty}=0$.

The application of models (6-8) leads to a description of the supercapacitor dynamics in the form of fractional differential equations. The problem of modelling supercapacitors with the application of fractional-order equations is presented e.g. in [14-16]. It was either based only on the arbitrarily-selected Cole-Davidson model of dielectric relaxation [14], or was not connected with such kind of a model at all. The authors of the paper examined the application of the Cole-Cole, Cole-Davidson, and integer order models, where in the last case, 2nd- and 3rd-order models were analysed. Their comparison is contained in, among others, [18] and Table 1.

The Cole-Cole model (7) application leads directly to the description of the impedance in the canonical fractional-order form [1]:

$$
Z(s)=\frac{b_{0} s^{\beta_{0}}+b_{1} s^{\beta_{1}}+\cdots+b_{m-1} s^{\beta_{m-1}}+b_{m} s^{\beta_{m}}}{a_{0} s^{\alpha_{0}} a_{1} s^{\alpha_{1}+\cdots+a_{n-1}} s^{\beta_{n-1}}+a_{n} s^{\alpha_{n}}}
$$

where exponents $\alpha_{k}$ and $\beta_{k}$ are real numbers. This paper is concerned mainly with this model because of its clarity. In the case of Cole-Davidson model (8), there is a binomial in the equation raised to a fractional power $(1+j \omega T)^{\gamma}$. To transform the impedance to form (9) on the basis of this model, a shift in the complex domain should be applied. If for the model (8) we assume shift $\alpha=1 / T$, then, after shifting, $(1+s T)^{\gamma}$ is replaced by $s^{\gamma}[14]$.

If in Fig. 2 the inductance is omitted, then the impedance of the analysed system can be presented as:

$$
Z_{c c}(s)=R_{c}+\frac{R_{u}}{1+s R_{u} C(s)}
$$

Table 1

Comparison of approximation accuracy of frequency and time responses of supercapacitors

\begin{tabular}{|c|c|c|c|c|c|c|c|c|}
\hline \multirow{2}{*}{ Capacity } & \multicolumn{7}{|c|}{ Standard deviation of approximation by model } \\
\cline { 2 - 10 } & \multicolumn{2}{|c|}{$2^{\text {nd }}$ order integer } & \multicolumn{2}{c|}{$3^{\text {nd }}$ order integer } & \multicolumn{2}{|c|}{ Cole-Cole } & \multicolumn{2}{c|}{ Cole-Davidson } \\
\hline Response & frequency & time & frequency & time & frequency & time & frequency & time \\
\hline $0.047 \mathrm{~F}$ & $9.8 \%$ & $11.2 \%$ & $5.3 \%$ & $6.3 \%$ & $5.1 \%$ & $5.1 \%$ & $3.5 \%$ & $2.7 \%$ \\
\hline $0.1 \mathrm{~F}$ & $10.5 \%$ & $16.4 \%$ & $4.4 \%$ & $4.9 \%$ & $4.8 \%$ & $2.3 \%$ & $3.8 \%$ & $2.7 \%$ \\
\hline $0.33 \mathrm{~F}$ & $16.3 \%$ & $22.6 \%$ & $7.8 \%$ & $7.3 \%$ & $7.8 \%$ & $2.6 \%$ & $5.4 \%$ & $3.4 \%$ \\
\hline $0.6 \mathrm{~F}$ & $13.7 \%$ & $11.4 \%$ & $10.0 \%$ & $8.8 \%$ & $10.4 \%$ & $9.9 \%$ & $14.0 \%$ & $3.9 \%$ \\
\hline $100 \mathrm{~F}$ & $8.0 \%$ & $4.4 \%$ & $5.8 \%$ & $5.8 \%$ & $6.2 \%$ & $6.2 \%$ & $6.0 \%$ & $6.0 \%$ \\
\hline $2700 \mathrm{~F}$ & $3.2 \%$ & $2.4 \%$ & $1.9 \%$ & $2.2 \%$ & $3.4 \%$ & $5.4 \%$ & $1.9 \%$ & $3.1 \%$ \\
\hline
\end{tabular}


As a result of transformations based on the Cole-Cole model, with the assumption that $\varepsilon_{\infty}=0$, the following is obtained:

$$
\mathrm{Z}_{\mathrm{CC}}(\mathrm{s})=\frac{\left(1+\frac{\mathrm{R}_{\mathrm{c}}}{\mathrm{R}_{\mathrm{u}}}\right)+\mathrm{s}^{\delta}\left(1+\frac{\mathrm{R}_{\mathrm{c}}}{\mathrm{R}_{\mathrm{u}}}\right) \mathrm{T}^{\delta}+\mathrm{sR}_{\mathrm{C}} \mathrm{C}}{\frac{1}{\mathrm{R}_{\mathrm{u}}}+\mathrm{s}^{\delta} \frac{\mathrm{T}^{\delta}}{\mathrm{R}_{\mathrm{u}}}+\mathrm{sC}}
$$

The measured amplitude-phase characteristics of supercapacitors versus frequency can be approximated by model (9) using the method of the least squares. The applied performance index takes the form of:

$$
J_{f}=\frac{1}{N-1} \sum_{i=1}^{N}\left(\frac{\left|z_{C C}\left(j \omega_{i}\right)-z_{p}\left(j \omega_{i}\right)\right|}{\left|z_{p}\left(j \omega_{i}\right)\right|}\right)^{2},
$$

where:

$\mathrm{Z}_{\mathrm{CC}}(\mathrm{j} \omega)$ - the impedance approximating the real impedance of the supercapacitor (11),

$\mathrm{Z}_{\mathrm{P}}(\mathrm{j} \omega)$ - measured impedance of the supercapacitor,

$\omega_{i}-$ angular frequency for $i^{\text {th }}$ measuring point.

This performance index can be treated as the variance of the approximation error. For example, the approximation of the characteristics of the Panasonic $0.33 \mathrm{~F}$ supercapacitor is illustrated by a graph in Fig. 3. The approximating impedance, determined on the basis of minimization of performance index (12), in this case is:

$$
\mathrm{Z}_{\mathrm{CC}}(\mathrm{s})=\frac{1.00+13.5 \mathrm{~s}^{0.674}+7.81 \mathrm{~s}}{1.65 \times 10^{-7}+2.23 \times 10^{-6} \mathrm{~s}^{0.674}+0.338 \mathrm{~s}},
$$

where the characteristic relaxation time constant $\mathrm{T}$ of a dielectric is $48 \mathrm{~s}$.

The measured frequency response of amplitude and phase is approximated by (13), with the performance index (12) equal to $7.8 \%$, while the Cole-Davidson model gives $5.4 \%$ (Fig. 3).
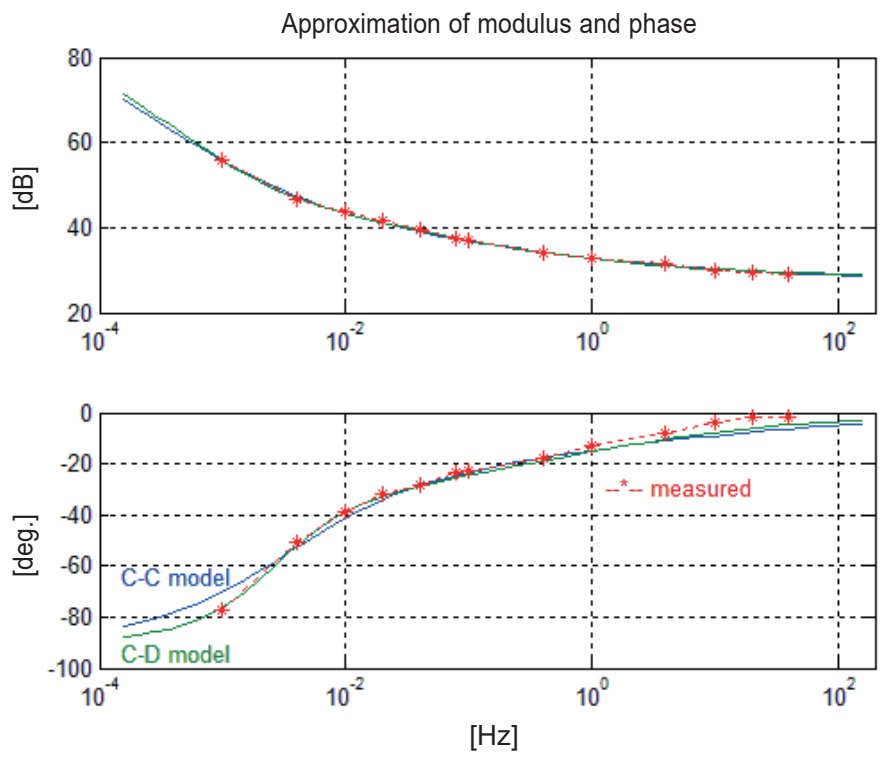

Fig. 3. The approximation of the measured frequency response of the $0.33 \mathrm{~F}$ supercapacitor (asterisks) by the fractional order transfer function based on the Cole-Cole and Cole-Davidson models
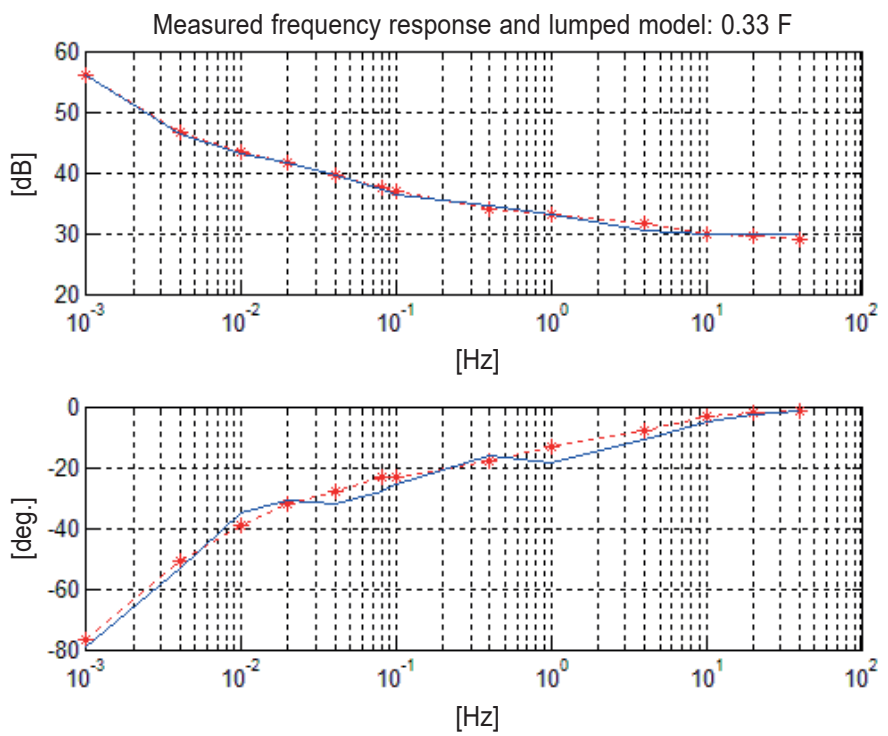

Fig. 4. The approximation of the measured frequency response of the $0.33 \mathrm{~F}$ supercapacitor (asterisks) by the lumped $3^{\text {rd }}$ integer order transfer function

It can be compared with integer-order models. For the second-order model, the performance index is $16.3 \%$, and for the third order it is $7.8 \%$ (Fig. 4). We also have investigated other supercapacitors. The results are shown in Table 1. It can be stated that the approximation on the basis of fractional-order models is better than those on the basis of the mentioned integer-order models [18]. Most often the results from the Cole-Davidson model were slightly better than those from the Cole-Cole model. When using the analysed models, the sequence of the mean square error levels of the time responses to rectangular current waveform (Table 1) were similar to those of the frequency response.

The example of time response to rectangular current waveform of $2 \mathrm{~s}$ period of the $0.33 \mathrm{~F}$ supercapacitor and its simulation on the basis of the described models is illustrated in Fig. 5. The approximation errors of time responses are presented in Fig. 6.

The choice of sufficient accuracy of the supercapacitors modelling was associated, among others, with the accuracy and stability of their parameters. For example, for a GS-130 supercapacitor of $2.4 \mathrm{~F} \pm 20 \%$ nominal capacity (made by CAP-XX), the equivalent serial resistance (ESR) at $-40^{\circ} \mathrm{C}$ is about 2.2 times larger than that at the room temperature and at $+70^{\circ} \mathrm{C}$ it is about $80 \%$ of that value [22]. So, the changes of the supercapacitor dynamic parameters are in the operating conditions relatively larger than inaccuracy of the model based on the Cole-Cole equation. In consequence, the choice of the Cole-Cole model of supercapacitor results from its form that is convenient for carrying out the intended analysis.

For an easier analysis of the dynamic phenomena in the supercapacitor, its impedance (11) can be divided into simple components. Since $R_{c}=23 \Omega$ and $R_{u}=600 \mathrm{k} \Omega$, then:

$$
R_{c} \ll R_{u}
$$




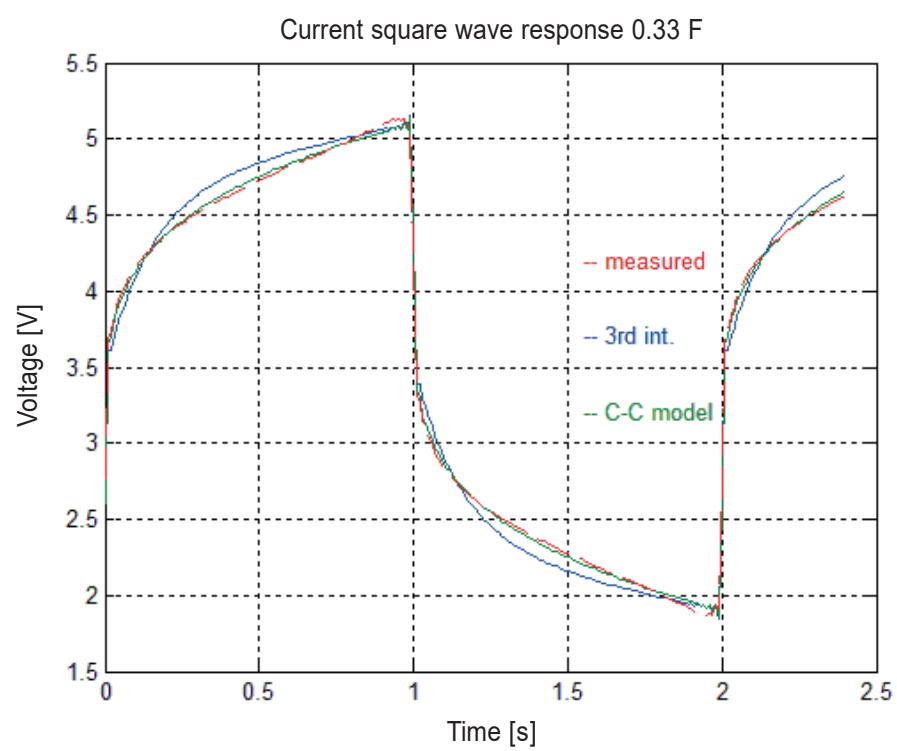

Fig. 5. The voltage time response of the $0.33 \mathrm{~F}$ supercapacitor to 0.5 $\mathrm{Hz}$ square waveform current and its simulation on the $3 \mathrm{rd}$ integer order and Cole-Cole models basis

and the approximating equation can be expressed as [17]:

$$
Z_{c c}(s)=Z_{c c 1}(s)+Z_{c c 2}(s)+Z_{c c 3}(s),
$$

where:

$$
\begin{aligned}
& Z_{c c 1}(s)=R_{c} \\
& Z_{c c 2}(s)=\frac{R_{u}}{1+s R_{u} C} \\
& Z_{c c 3}(s)=\frac{s^{\partial} T^{\partial} R_{u}}{1+s R_{u} C}
\end{aligned}
$$

Where the $\mathrm{Z}_{\mathrm{CC} 1}$ component is the equivalent serial resistance, associated mainly with the resistance of porous electrodes [11], $\mathrm{Z}_{\mathrm{CC} 2}$ is the typical impedance of the capacitor with the leakage resistance, and $\mathrm{Z}_{\mathrm{CC} 3}$ characterizes the relaxation phenomenon and is described by a fractional order equation.

Figure 7 presents a double-logarithmic Bode graph of the impedance modulus $Z_{C C}(\omega)$. The graph also shows the asymptotes associated with components $(16 \mathrm{a}-16 \mathrm{c})$. This diagram does not include the very low frequency range, in which the slope of the characteristics changes due to the influence of the leakage resistance $R_{U}$. This effect is related to the time constant $R_{U} C$ with a value typically above $10^{5} \mathrm{~s}$, whose magnitude is several orders higher than the characteristic time constant of dielectric relaxation.

The slope of the asymptotes at the Bode diagram (Fig. 7) depends on the order of the impedance of $\mathrm{Z}_{\mathrm{CC}}$ components. For the presented frequency range, the slope of the $\mathrm{Z}_{\mathrm{CC} 1}$ asymptote is zero and the slope of $Z_{\mathrm{CC} 2}$ asymptote is $-20 \mathrm{~dB} / \mathrm{decade}$. In the case of $Z_{\mathrm{CC} 3}$ component, which is fractional order, the slope of the asymptote is $(1-\partial) \times(-20 d B) /$ decade. It's worth noticing

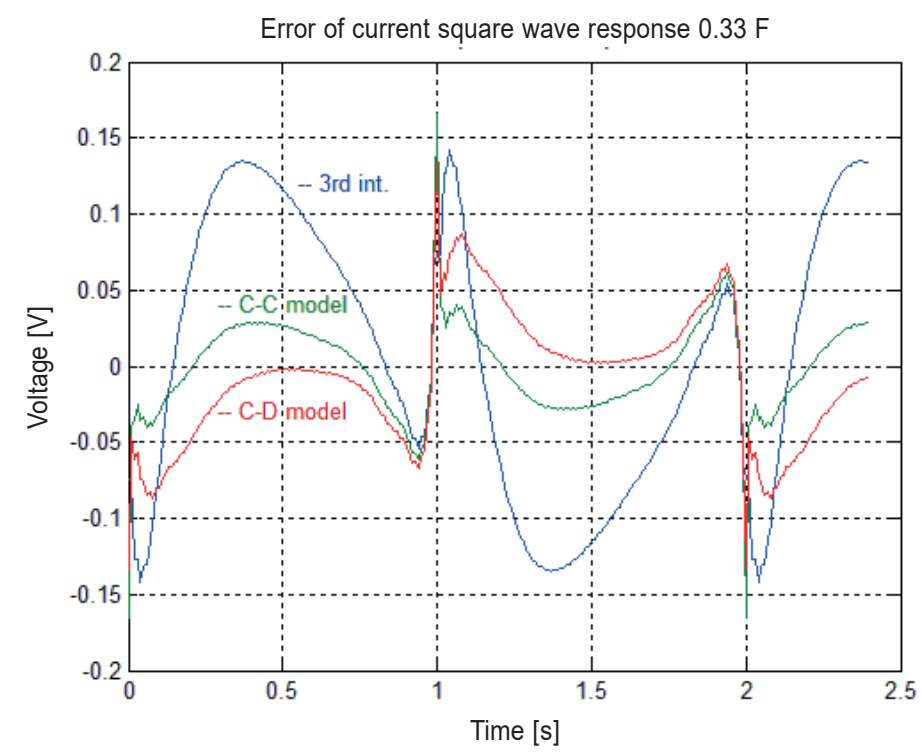

Fig. 6 . The errors of time response approximation by $3^{\text {rd }}$ integer order and Cole-Cole and Cole-Davidson fractional order models

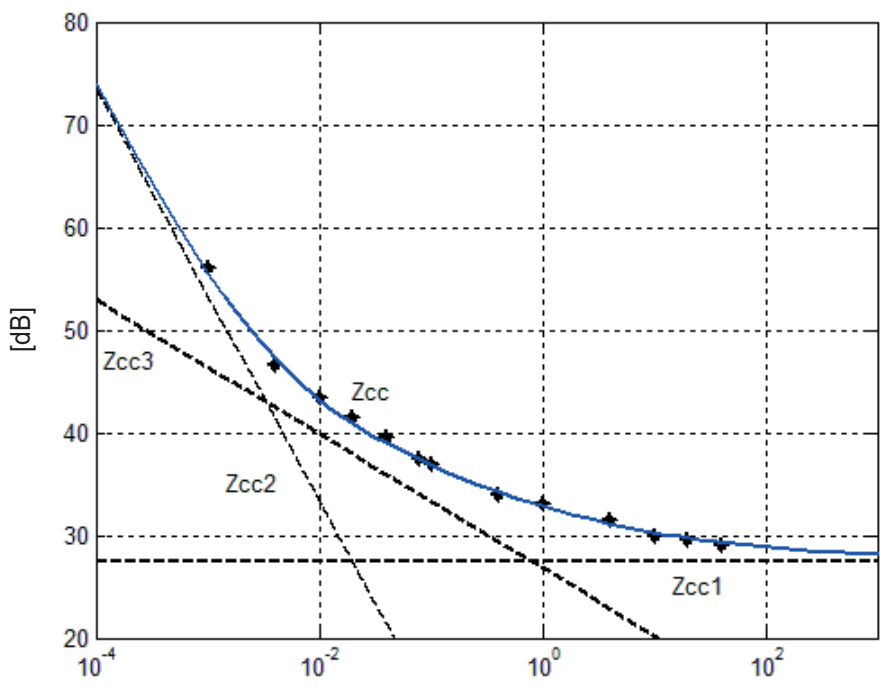

$[\mathrm{Hz}]$

Fig. 7. The component moduli of impedances (26) of the $0.33 \mathrm{~F}$ supercapacitor

that the coefficient $\delta$ is the parameter of the dielectric relaxation phenomenon in the Cole-Cole model (7).

Voltage response ${ }_{V C C}(t)$ of the supercapacitor to the step of charging or discharging current can be compared with the response of the model of a capacitor in the form of an ideal capacitor connected in series with a resistance. The response of this model after an initial step is a linear function of time. The dielectric relaxation causes the initial phase of the supercapacitor response ${ }_{V C C}(t)$ to differ significantly from the mentioned linear change of the reference model response. 
0.008 A current step response, Cole-Cole model, $0.33 \mathrm{~F}$

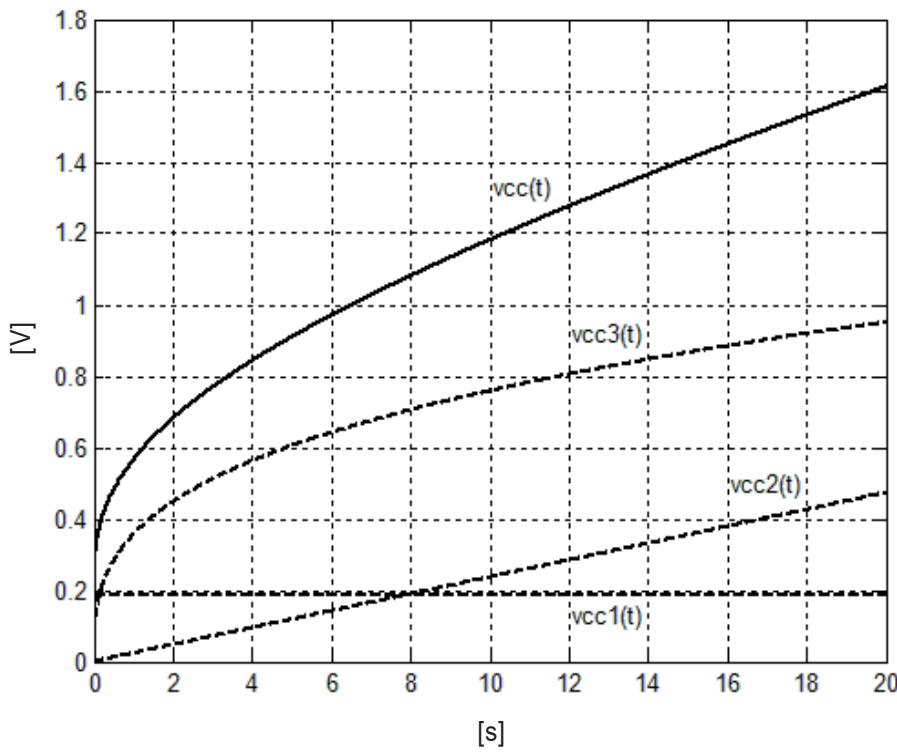

Fig. 8. Step responses $\mathrm{v}_{\mathrm{CC}}(\mathrm{t})$ of impedance (8) and its components $(27 \mathrm{a}-27 \mathrm{c})$ due to $8 \mathrm{~mA}$ step excitation

Response $v_{C C}(t)$ can be considered as the sum of responses $v_{C C 1}, v_{C C 2}$, and $v_{C C 3}$, which can be attributed respectively to components $Z_{C C 1}, Z_{C C 2}$, and $Z_{C C 3}$ (13). For the supercapacitor characteristics shown in Fig. 3 the simulated response to charging with an $8 \mathrm{~mA}$ current is presented in Fig. 8. As can be seen, the initial response nonlinearity $v_{C C}(t)$ is determined by the $v_{C C 3}(t)$ response of the component of fractional order. The duration of the significant influence of this component on the non-linearity of the total response is comparable to the characteristic time constant $T$ of dielectric relaxation.

The measurements of various types of supercapacitors have shown that not always is the dielectric relaxation influence on the dynamic characteristic impedance of the same significance. This effect could be neglected if, for example, the equivalent serial resistance $R_{C}$ was, as shown in Fig. 8, larger. Then, correspondingly to the $Z_{C C 1}$ asymptote, it would be located higher on the diagram and, together with $Z_{C C 2}$, it would dominate over the asymptote $Z_{C C 3}$.

The above considerations are based on the description of the supercapacitor impedance by the fractional-order model. The conclusions concerning the shape and time constant of voltage response to the current step will be used for the analysis of the capacitance measurement conditions according to the IEC 62391-1:2006 standard.

\section{The measurement of the supercapacitor capacitance according to the IEC standard}

The determination of the supercapacitors capacitance value according to the IEC 62391-1:2006 "Fixed electric double-layer capacitors for use in electronic equipment" standard [21] is based on the process of the capacitor discharging with a con-

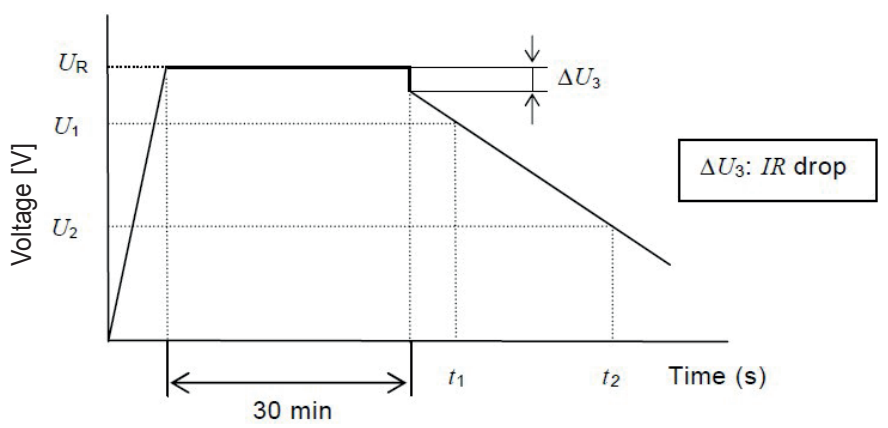

Fig. 9. Voltage on the capacitor terminals during the capacitance measurements according to the IEC 62391-1:2006 standard [21]

stant current. The graph in the description of the standard, illustrating the procedure of the initial charging to the nominal voltage $U_{\mathrm{R}}$ and discharging, is presented in Fig. 9.

According to the standard, the determination of capacitance $\mathrm{C}$ of a supercapacitor bases on the equation [21]:

$$
C=\frac{I \times\left(t_{2}-t_{1}\right)}{U_{1}-U_{2}}
$$

where:

C - the capacitance $[\mathrm{F}]$,

I - the discharging current [A],

$\mathrm{U}_{1}$ - the measurement starting voltage $[\mathrm{V}]$,

$\mathrm{U}_{2}$ - the measurement end voltage [V],

$t_{1}$ - the time from discharge start to reaching $U_{1}[\mathrm{~s}]$,

$t_{2}$ - the time from discharge start to reaching $U_{2}[s]$,

The measurement conditions depend on the qualification of the supercapacitor to the application class. These conditions are presented in Table 2 [21].

"Note a" to the conditions presented in Table 2 admits the possibility of reduction of the discharge current value, even tenfold, in cases where the increment $\Delta \mathrm{U}_{3}$ exceeds $5 \%$ of $\mathrm{U}_{\mathrm{R}}$. However, this brings the conditions of the measurement to lower class, which raises doubts about the idea of the classification.

Table 2

The discharge conditions according to the IEC 62391-1:2006 standard [21]

\begin{tabular}{|c|c|c|c|c|}
\hline Classification & Class 1 & Class 2 & Class 3 & Class 4 \\
\hline Application & $\begin{array}{c}\text { Memory } \\
\text { backup }\end{array}$ & $\begin{array}{c}\text { Energy } \\
\text { storage }\end{array}$ & Power & $\begin{array}{c}\text { Instantaneous } \\
\text { power }\end{array}$ \\
\hline Charge time & 30 min. & 30 min & 30 min & 30 min \\
\hline $\mathrm{I}(\mathrm{mA})$ & $1 \times \mathrm{C}$ & $0.4 \times \mathrm{CU}_{\mathrm{R}}$ & $4 \times \mathrm{CU}_{\mathrm{R}}$ & $40 \times \mathrm{CU}_{\mathrm{R}}$ \\
\hline $\mathrm{U}_{1}$ & \multicolumn{2}{|c|}{ The value to be $80 \%$ of charging voltage $\left(0.8 \times \mathrm{U}_{\mathrm{R}}\right)$} \\
\hline $\mathrm{U}_{2}$ & \multicolumn{3}{|c|}{ The value to be $40 \%$ of charging voltage $\left(0.4 \times \mathrm{U}_{\mathrm{R}}\right)$} \\
\hline
\end{tabular}

NOTE: a) If $\Delta \mathrm{U}_{3}$ exceeds $5 \%\left(0.05 \times \mathrm{U}_{\mathrm{R}}\right)$ of the charging voltage in the initial characteristics, the current value may be reduced by one half, one fifth, or one tenth. (...) 
The conditions for discharging the capacitor in order to determine its capacitance (Table 2) can also be characterized in another way. For an ideal capacitor, characterized only by the capacitance $\mathrm{C}$, the time of complete discharge and times $t_{1}$ and $t_{2}$ would, under these conditions, have the values shown in Table 3.

Table 3

Times of an ideal capacitor discharging under the conditions of IEC 62391-1:2006 standard

\begin{tabular}{|c|c|c|c|c|}
\hline Classification & Class 1 & Class 2 & Class 3 & Class 4 \\
\hline Application & $\begin{array}{c}\text { Memory } \\
\text { backup }\end{array}$ & $\begin{array}{c}\text { Energy } \\
\text { storage }\end{array}$ & Power & $\begin{array}{c}\text { Instantaneous } \\
\text { power }\end{array}$ \\
\hline $\begin{array}{c}\text { Time of full } \\
\text { discharge }\end{array}$ & - & $2500 \mathrm{~s}$ & $250 \mathrm{~s}$ & $25 \mathrm{~s}$ \\
\hline $\mathrm{t}_{1}$ & - & $500 \mathrm{~s}$ & $50 \mathrm{~s}$ & $5 \mathrm{~s}$ \\
\hline $\mathrm{t}_{2}$ & - & $1500 \mathrm{~s}$ & $150 \mathrm{~s}$ & $15 \mathrm{~s}$ \\
\hline
\end{tabular}

As mentioned, the typical characteristic relaxation time constants in supercapacitors have values from a few to tens of seconds [11], and they are of the same order as the discharging times of the capacitor during the measurement shown in Table 3 for Class 4. Due to the possibility of the occurrence of a significant response non-linearity during the measurement, this phenomenon can have a considerable influence on the result of the determined capacitance of supercapacitors.

The standard also allows alternative capacitance measurement of the supercapacitor $\mathrm{C}$ connected via resistor $\mathrm{R}$ to the voltage source $U_{R}$. The time constant $\tau=R C$ is determined on the basis of exponential curve $u_{c}(t)$, which, by definition, corresponds to the charging of an ideal capacitor from $u_{c}(0)=0$ :

$$
u_{c}(t)=U_{R}\left(1-e^{-t / \tau}\right)
$$

Time constant $\tau$ is determined as $\mathrm{t}=\tau$, at the voltage level:

$$
u(\tau)=U_{R}\left(1-e^{-1}\right)=0.632 U_{R}
$$

Without any remark, the standard recommends $0.632 \mathrm{U}_{\mathrm{R}}$ level and the conditions at which $60 \mathrm{~s} \leq \tau \leq 120 \mathrm{~s}$ is fulfilled.

It should be emphasized that the manufacturers of supercapacitors declare the values of nominal capacitance without taking into account the classes of applications listed in the standard and without specification of the measurement conditions.

The effect of dielectric relaxation does not occur significantly in all types of supercapacitors. An example of capacitance measurement under Class 4 conditions with the result close to the nominal capacitance is illustrated in Fig. 10. The points $\left(\mathrm{U}_{1}, \mathrm{t}_{1}\right)$ and $\left(\mathrm{U}_{2}, \mathrm{t}_{2}\right)$ at constant current discharge diagrams in Fig. 10 and next figures are marked with asterisks. The discharging under Class 4 conditions of a Maxwell HC series

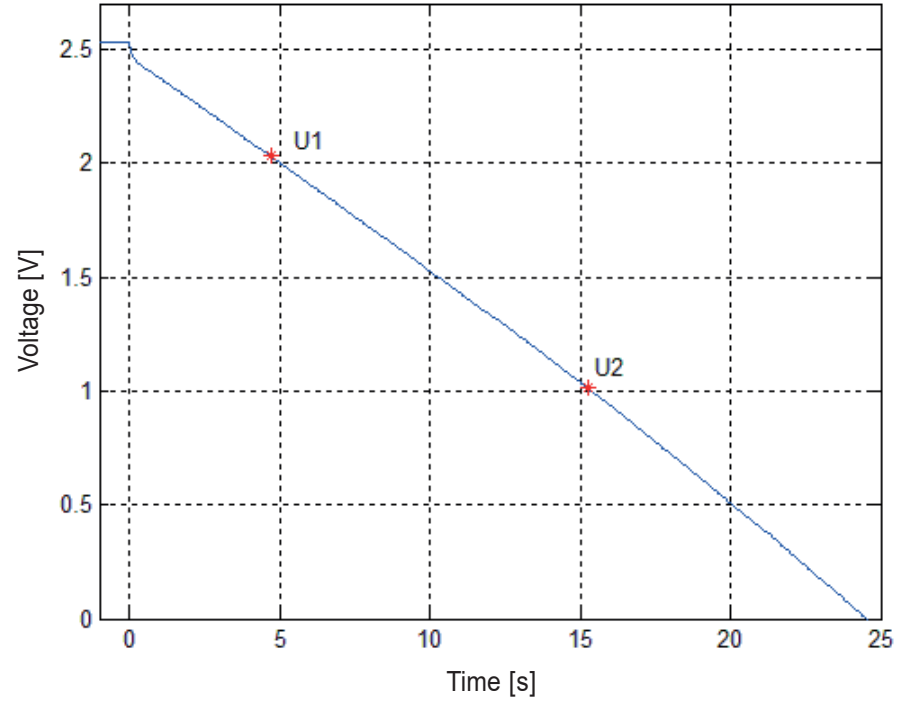

Fig. 10. The discharge of the supercapacitor of $10 \mathrm{~F}$ nominal with $1 \mathrm{~A}$ current (Class 4): the measured capacitance according to the IEC Standard is equal $10.3 \mathrm{~F}$

capacitor of $10 \mathrm{~F}$ nominal value is shown in Fig. 10. In this case, the result of the measurement is $\mathrm{C}=10.8 \mathrm{~F}$. Under the measurement conditions provided for Class 3 , the result is $\mathrm{C}=10.3 \mathrm{~F}$. In both cases, the measured capacitance does not exceed $30 \%$ of the tolerance limits for this type of supercapacitors.

An example of a significant influence of dielectric relaxation on the measurement results can be presented on the basis of the capacitance measurement in Class 4 conditions of Panasonic Gold Capacitor of nominal capacitance $1 \mathrm{~F}$. This process is illustrated in Fig. 11.

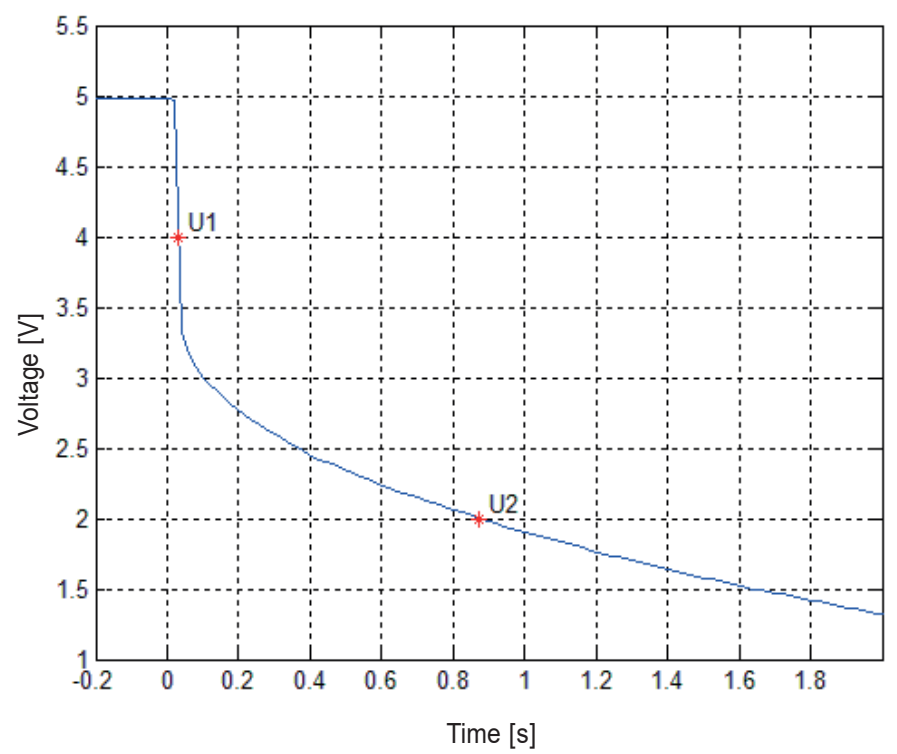

Fig. 11. The discharge of the supercapacitor of $1 \mathrm{~F}$ nominal capacitance with $200 \mathrm{~mA}$ current (Class 4): the measured capacitance according to the IEC Standard is $0.08 \mathrm{~F}$ 
The result obtained on the basis of the determination of the $U_{1}$ and $U_{2}$ voltage levels is more than ten times smaller than the nominal capacitance, and it is $0.08 \mathrm{~F}$.

For obvious reasons, this is not a correct measurement, so the recommendation of measurement current reduction should be applied. The right result of $1.1 \mathrm{~F}$ value can be obtained for the Class 3 conditions of the standard, at 1/10th of the recommended current. During the measurement in Class 4 conditions, the time between $\mathrm{U}_{1}=4 \mathrm{~V}$ and $\mathrm{U}_{2}=2 \mathrm{~V}$ levels is only $0.8 \mathrm{~s}$, while in Class 3 conditions it is $110 \mathrm{~s}$. The differences are due to the relaxation phenomena, as well as the serial resistance $R_{c}$. For comparison of the two cases, in Fig. 12 the graph of the dynamic changes of supercapacitor voltage of this supercapacitor vs. the supplied electric charge $\mathrm{Q}$ is presented.

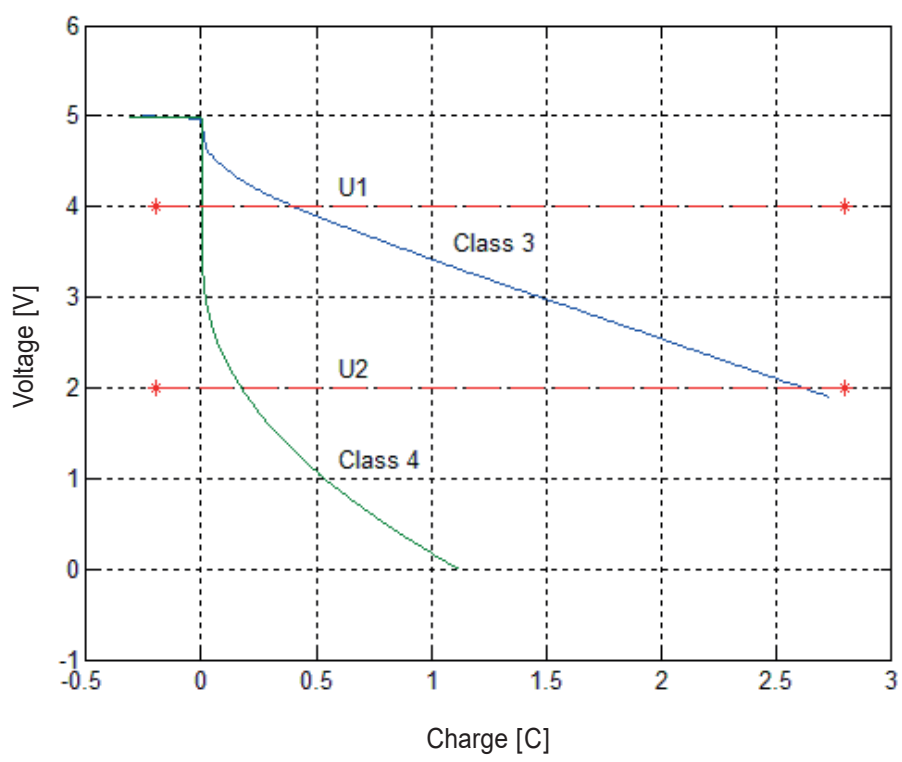

Fig. 12. The comparison of dynamic changes of $1 \mathrm{~F}$ capacitor voltage vs. charge at $20 \mathrm{~mA}$ current (Class 3): the measurement result is $1.1 \mathrm{~F}$ and at $200 \mathrm{~mA}$ (Class 4) it is $0.08 \mathrm{~F}$

The effect of the measurement based on the non-linear part of the voltage curve shown in Fig. 11 is close to the extreme case. In other cases, the result of the measurement can differ not so much from the nominal value of the capacitance, and the remark "the current value may be reduced" (Table 2) in the standard can be ignored, because it is not formulated as a requirement.

According to the authors, the capacitance measurement conditions described in the standard have not sufficiently taken into consideration the dielectric relaxation phenomenon. The modelling of supercapacitor dynamics treated as black boxes often leads to general conclusions. A particularly dubious recommendation of the standard is the abovementioned possibility of capacitance measurement in an RC circuit connected to a DC voltage source. The occurrence of the $\mathrm{Z}_{\mathrm{CC} 3}$ component in supercapacitor impedance (15) can greatly change the shape of the time response in respect to the exponential curve obtained during the charging of an ideal capacitor. Furthermore, such an approach omits the voltage drop on serial resistance $\mathrm{R}_{\mathrm{C}}$, which is the $\mathrm{Z}_{\mathrm{CC} 1}$ component of impedance (15).

In conclusion, it can be stated that the IEC 62391-1:2006 standard recommends the current value at which the measurement duration is similar to the time constant of the supercapacitor dielectric relaxation. Such is the case for Class 4 of supercapacitor application named "instantaneous power". The use of this recommendation often leads to a result even several times lower than that of the nominal capacitance value - confirmed by measurements according to the terms of a lower class of this standard. These results can't therefore be the main base for e.g. power supply calculations. In addition, it should be noted that the manufacturers of supercapacitors do not attribute their products to the classes mentioned in the standard and specify only the nominal capacitances without any stipulations. On the other hand, it may be very important for both the designer and the user to know the properties of a given type of supercapacitor under the conditions of high dynamic load similar to Class 4 conditions of capacitance measurement.

Therefore, the authors suggest for Class 4 to take the measurements of the nominal capacitance in heavier conditions, e.g. such as those for Class 3 , and to introduce an additional new term of dynamic capacitance determined for high load conditions. The dynamic capacitance would be determined under the conditions currently provided for Class 4 of the standard. The comparison of the nominal capacitance to the value defined as the dynamic capacitance would allow for determining the effectiveness of the use of considered type of supercapacitors under heavy load.

In the case of introduction of the concept of dynamic capacitance, it would be worthwhile to consider giving up the division into the classes of applications.

\section{Conclusions}

Description of the dynamics by differential equations of fractional order is most effective for certain kinds of systems. These include supercapacitors, where the influence of dielectric relaxation is significant. The model of dynamics expressed by the fractional-order differential equations can be directly associated with the equations describing the physical phenomenon in question. Absence of such relationship can lead to the acceptance of a black box model, which, by coincidence, does not sufficiently describe the dynamics of the whole group of characterized objects.

An example of formulation of such partly improper and imprecise measurement conditions can be the IEC 62391-1:2006 standard, in which it is recommended, in individual cases, the possibility of changing these conditions. This can significantly affect the measurement results. The conclusions based on the model of the supercapacitor impedance of fractional-order presented here allow the rationalization of measurement requirements. 


\section{REFERENCES}

[1] C.A. Monje, Y. Chen, B.M. Vinagre, D. Xue, and V. Feliu-Batlle, Fractional-Order Systems and Controls: Fundamentals and Applications, Springer-Verlag, London, 2010.

[2] S. Guermah, S. Djnnoune, and M. Bettayeb, Advances in Discrete-Time Systems. Chapter 8: Discrete-Time Fractional-Order Systems: Modelling and Stability, Intech, 2012.

[3] I. Petras, B.M. Vinagre, L. Dorcak, and V. Feliu, "Fractional digital control of a heat solid: Experimental results", Proceedings of the International Carpathian Control Conference, 365-370 (2002).

[4] C.M.A. Brett and A.M. Oliveira-Brett, Electrochemistry. Principles, Methods, and Applications, Oxford University Press, Oxford, 1993.

[5] Y.Q. Chen, I. Petras, and D. Xue, "Fractional order control - A tutorial”, 2009 American Control Conference, ACC '09, (2009).

[6] L. Shi, M.L. Crow, "Comparison of ultracapacitor electric circuit models", 2008 IEEE Power and Energy Society General Meeting - Conversion and Delivery of Electrical Energy in the 21st Century, (2008).

[7] G.A. Badea, "Supercapacitors - the batteries of future", Bulletin Scientifique en Langues Étrangères Appliquées 3, http://revues-eco.refer.org/BSLEA/index.php?id=491, (2015).

[8] R. Martin, J.J. Quintana, A. Ramos, and I. de la Nuez, "Modeling electrochemical double layer capacitor, from classical to fractional impedance", Journal of Computational and Nonlinear Dynamics 3 (2), 61-66 (2008).

[9] H. Göhr, "Impedance modelling of porous electrode", Electrochemical Applications 1, 2-3 (1997).

[10] X. Yang, C. Cheng, Y. Wang, L. Qiu, and D. Li, "Liquid-mediated dense integration of graphene materials for compact capacitive energy storage", Science 341 (6145), 534-537 (2013).

[11] R. Farma, M. Deraman, A. Awitdrus, I.A. Talib, R. Omar, J.G. Manjunatha, M.M. Ishak, N.H. Basri, and B.N.M. Dola, "Physical and electrochemical properties of supercapacitor electrodes derived from carbon nanotube and biomass carbon", International Journal of Electrochemical Science 8, 257-273 (2013).
[12] N. Bertrand, J. Sabatier, O. Briat, and J.-M. Vinassa, "Fractional non-linear modeling of ultracapacitors", Communications Nonlinear Science and Numerical Simulation 15 (5), 1327-1337 (2010).

[13] J.-L. Déjardin and J. Jadzyn, "Determination of the nonlinear dielectric increment in the Cole-Davidson model", The Journal of Chemical Physics 125 (11), 114503 (2006).

[14] A. Dzieliński, G. Sarwas, and D. Sierociuk, "Comparison and validation of integer and fractional order ultracapacitor models", Advances in Difference Equations 2011:11, (2011)

[15] T.J. Freeborn, B. Moundy, and A.S. Elwakil, "Measurement of supercapacitor fractional-order model parameters from voltage-excited step response", IEEE Journal on Emerging and Selected Topics in Circuits and Systems 3 (3), 367-376 (2013).

[16] N. Maim, D. Isa, and R. Arelhi, "Modelling of ultracapacitor using a fractional-order equivalent circuit", International Journal of Renewable Energy Technology 6 (2), 142-163 (2015).

[17] M. Orzyłowski and M. Lewandowski, "Computer modeling of supercapacitor with Cole-Cole relaxation model", Journal of Applied Computer Science Methods 5 (2), 105-121 (2013).

[18] M. Lewandowski and M. Orzyłowski, "The application of fractional calculus for supercapacitor dynamics modeling", Przegląd Elektrotechniczny 90 (8), 13-17 (2014), [in Polish].

[19] A. Szeląg and T. Maciołek, "A 3 kV DC electric traction system modernisation for increased speed and trains power demand - problems of analysis and synthesis", Przegląd Elektrotechniczny 89 (3a), 21-28 (2013).

[20] M. Wieczorek and M. Lewandowski, "Mathematical representation of an energy management strategy for hybrid energy storage system in electric vehicle and real time optimization using a genetic algorithm", Applied Energy 192, 222-233 (2017).

[21] International Standard IEC 62391-1:2006. Fixed Electric Double-Layer Capacitors for Use in Electronic Equipment. Part 1: Generic Application, IEC, 2006.

[22] GS130/GS230 Supercapacitor Datasheet V4.1, CAP-XX, 2015. 